\title{
Editorial: Everyday Virtual and Augmented Reality: Methods and Applications
}

\author{
Christoph W. Borst ${ }^{1 *}$, Benjamin Weyers ${ }^{2}$, Adalberto L. Simeone ${ }^{3}$, Arindam Dey $^{4}$ and \\ Daniel Zielasko ${ }^{2}$ \\ ${ }^{1}$ Center for Advanced Computer Studies, University of Louisiana at Lafayette, Lafayette, LA, United States, ${ }^{2}$ Department of \\ Computer Science, University of Trier, Trier, Germany, ${ }^{3}$ Department of Computer Science, KU Leuven, Leuven, Belgium, \\ ${ }^{4}$ Department of Computer Science, The University of Queensland, Brisbane, QLD, Australia
}

Keywords: everyday virtual reality, everyday augmented reality, social virtual reality, consumer virtual reality, casual virtual reality, commodity virtual reality, ergonomics, field studies

Editorial on the Research Topic

Everyday Virtual and Augmented Reality: Methods and Applications

\section{INTRODUCTION}

Recent improvements in affordable virtual and augmented reality technology enable broader adoption in everyday contexts such as homes, schools, and health care settings. This Frontiers Research Topic presents articles that give insight into the everyday use of VR/AR or that provide techniques for interface improvements.

Three articles report field studies of everyday applications. Studying VR and AR in everyday contexts provides practical insight into real-world deployments, with a level of ecological validity beyond artificial laboratory environments. Two articles discuss input methods that support improved interaction with minimal added cost (sketching, whole-hand interaction). Two Opinion and Perspective articles discuss considerations for older adults and for molecular science students.

\section{FIELD STUDIES OF EVERYDAY VR}

Pimentel et al. study the use of VR to mitigate pain during tattooing, using the Ocean Rift underwater environment with Oculus headsets. The authors note that the tattooing context offers certain benefits for VR pain studies compared to most prior contexts: the mechanism inducing pain is relatively consistent and pain is experienced for a long duration in a manner that allows several rating intervals. A main finding is that, although early pain ratings were higher than those of a control group, reported pain reduced for a time, until headset removal. Practical aspects are discussed for VR during tattooing. An underwater or zero-gravity VR environment is suggested to match the various body positions involved, and the compatibility of VR with various body positions is described. Other suggestions include avoiding VR for tattoo locations that may trigger reflexive responses and for first-time tattoo recipients who may require careful monitoring.

The two articles below investigated everyday networked VR to support virtual meetings, in the context of conference and class meetings that switched to a remote format due to SARS-CoV-2. Both studies included the Mozilla Hubs social VR tool for attendance by home VR users. Both studies show a positive potential of networked VR meetings, but they also reveal substantial limitations to be addressed by future everyday VR technology. 
Ahn et al. considered multiple delivery methods of the IEEE VR 2020 conference, one of the first academic conferences to switch to a completely remote format. Surveys and field observations were used to evaluate results. The remote format led to a high number of registrations and attendee diversity enabled by factors such as reduced travel costs and reduced demand on family caregivers. Mozilla Hubs ratings showed increased social presence over non-VR alternatives. However, various practical and technical problems were encountered with Hubs. Headset removal for tasks such as keyboard use or due to fatigue led to reduced headset use later in the conference. Most Hubs users chose to view the environment on a standard monitor, despite many owning VR headsets. Audio and network problems were also reported. Hubs was most successful for social activities such as "Birds of a Feather" meetings, and less so for viewing talks and asking questions to presenters. Some attendee responses suggested that joining conversations was easier than at real physical venues, and this may relate to reduced cues about status and affiliations. Nonetheless, many attendees were using other meeting platforms, like Twitch, at the end of the conference.

Yoshimura and Borst studied a remote class with university students in Hubs and similarly found both promising aspects and difficulties for everyday networked VR. The use of headsets increased reported presence compared to viewing on standard monitors. Key ratings for headset VR varied widely and in high correlation to reported sickness symptoms that mainly reflected general discomfort. Some students removed headsets due to discomfort or for external tasks. Most students reported some audio or video glitches, and about half encountered some external distraction. In their final reflections on VR and remote methods used in other classes, students ranked VR highly overall in terms of motivation and sense of belonging to a university. Some students appreciated reduced anxiety or reduced visibility of using VR instead of video.

\section{INPUT AND INTERACTION}

Kern et al. extend a common handheld controller to support writing and sketching in VR and AR. This capability could enhance applications like the networked VR meetings above, enabling attendees to take notes or presenters to better annotate content without shifting to external tools or additional devices. Considering various controllers and tips for stylus-type interaction with a precision hand grip, the authors mounted the tip of an Apple Pencil on an Oculus Controller, without requiring any additional trackers, microcontrollers, or 3D printed connectors. They developed methods and open-source software for calibration and surface interactions, with promising initial results based on user evaluations. The authors suggest future integration with a commercial web-based plugin for generating, storing, and sharing sketches.
Isaac et al. present a plausibility-based filter to improve hand tracking. Optical hand tracking is increasingly appearing in everyday VR configurations, for example, using headset-embedded cameras of HTC Vive or Oculus Quest devices. The proposed filter adjusts joint angles to be closer to anatomically feasible values when they are detected out-ofbounds with respect to anatomy literature. A baseline handtracking neural network, with the filter included during training, is shown to perform well relative to two prior models. The filter can also reduce errors when added to output of the trained prior models, for certain settings (moderate correction level).

\section{OPINIONS AND PERSPECTIVES}

Seifert and Schlomann briefly outline beneficial uses of everyday VR and AR for older adults. They then call for more effort and investment to understand and support the needs of this population, noting a wide variation in skill levels, attitudes, living arrangements, etc. Two suggested methods for improvement are to involve older adults in participatory design and to offer them more support for developed tools.

Reen et al. survey considerations for educational VR in the context of molecular science education. They note that more work is needed to make good use of VR's hands-on experiential capabilities to communicate abstract theoretical concepts rather than just simulating lab activities. The authors present an initial roadmap and relate application and implementation aspects to mechanisms of learning.

\section{AUTHOR CONTRIBUTIONS}

All authors of this Editorial contributed substantially as editors of papers in this Special Topic. CB drafted this editorial with suggestions and approval from $\mathrm{BW}, \mathrm{AS}, \mathrm{AD}$, and $\mathrm{DZ}$.

Conflict of Interest: The authors declare that the research was conducted in the absence of any commercial or financial relationships that could be construed as a potential conflict of interest.

Publisher's Note: All claims expressed in this article are solely those of the authors and do not necessarily represent those of their affiliated organizations, or those of the publisher, the editors and the reviewers. Any product that may be evaluated in this article, or claim that may be made by its manufacturer, is not guaranteed or endorsed by the publisher.

Copyright (C) 2021 Borst, Weyers, Simeone, Dey and Zielasko. This is an open-access article distributed under the terms of the Creative Commons Attribution License (CC $B Y$ ). The use, distribution or reproduction in other forums is permitted, provided the original author(s) and the copyright owner(s) are credited and that the original publication in this journal is cited, in accordance with accepted academic practice. No use, distribution or reproduction is permitted which does not comply with these terms. 